\title{
Classification Airbrone LiDAR Point cloud Data
}

\author{
Naga Madhavi lavanya Gandi \\ Andhra University, Visakhapatnam, India
}

To Cite this Article

Naga Madhavi lavanya Gandi, "Classification Airbrone LiDAR Point cloud Data”, International Journal for Modern Trends in Science and Technology, Vol. 07, Issue 01, January 2021, pp.-36-39.

\section{Article Info}

Received on 22-November-2020, Revised on 18-December-2020, Accepted on 22-December-2020, Published on 28-December-2020.

\section{ABSTRACT}

Land cover classification information plays a very important role in various applications. Airborne Light detection and Ranging (LiDAR) data is widely used in remote sensing application for the classification of land cover. The present study presents a Spatial classification method using Terrasoild macros. The data used in this study are a LiDAR point cloud data with the wavelength of green:532nm, near infrared:1064nm and mid-infrared-1550nm and High Resolution RGB data. The classification is carried in TERRASCAN Module with twelve land cover classes. The classification accuracies were assessed using high resolution RGB data. From the results it is concluded that the LiDAR data classification with overall accuracy and kappa coefficient $85.2 \%$ and 0.7562 .

KEYWORDS: Airborne, LIDAR, TERRASOILD, spatial classification.

\section{INTRODUCTION}

With the increasing demand for basic human needs, the information of land use and land cover is important. This information of land cover classification is used for effective selection, planning and implementation of land use schemes[1]. Recent advancement in remote sensing technology used to collects both aerial and satellite data in repetitive basis and the remote sensing information together with GIS helps to generate various models of land cover classification. LiDAR is one of the advanced data sets used for the land cover classification. Light detection and ranging (LiDAR) shows enhanced spatial properties (elevation) [2]. The elevation information is used separate various land cover features [3]. For the classifications of LiDAR data studies have been conducted. For the classification of LiDAR point cloud data mostly use two types of classification techniques object-based classification and supervised classification. In object based classification the spatial parameters like elevation, size, shape etc., [4] and threshold of indices were used[5]. In supervised classification the LiDAR point cloud data was segmented and classified using supervised classification algorithms like support vector machine[6], k-NN algorithm[7], artificial neural network classifier[8] are used. In this study classification is done using Terrasoild (Terrascan, Terrascan, Terraphoto, and Terramodel).

\section{DATA AND STUDY AREA}

Data

In order to reconstruct both a three-dimensional model and a segmentation of semantic classes for an urban scene a data fusion contest has been organized by the Image Analysis and Data Fusion Technical Committee (IADFTC) of the IEEE Geosciences and Remote Sensing Society (GRSS), the Johns Hopkins University (JHU), and the 
Intelligence Advanced Research Project Activity (IARPA). In Data Fusion Contest 2018 are acquired by National Center for Laser Mapping, NCALM on February 16, 2017 between 16:31 and 18:18 GMT. The sensors that are used in the acquiring data are an Optech Titan MW (14SEN/CON340) with integrated camera (a LIDAR sensor operating at three different laser wavelengths), A DiMAC ULTRALIGHT+ a very high-resolution color imager with $70 \mathrm{~mm}$ focal length, An ITRES CASI 1500 (a Hyperspectral imager).

\section{Study area}

The study area that is taken for the present study is part of Houston city which is in the U.S. state of Texas. It is in southeast Texas near Galveston Bay and the Gulf of Mexico.

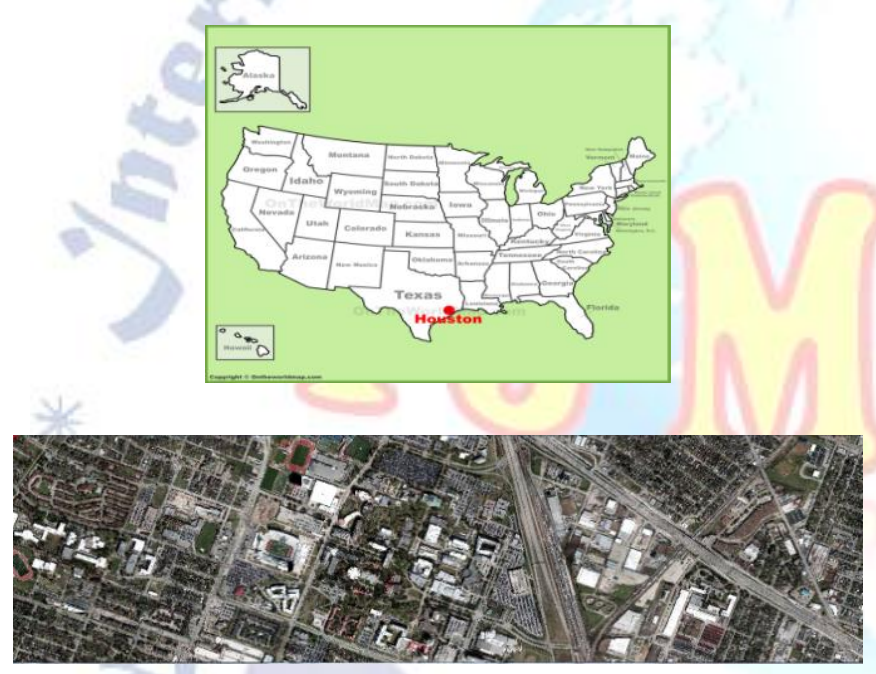

Figure.1., Study Area

The data used in this study are Multispectral-LiDAR point cloud data at $1550 \mathrm{~nm}$, $1064 \mathrm{~nm}$, and $532 \mathrm{~nm}$; Intensity raster from first return per channel and DSMs at a 50-cm GSD, Hyperspectral data covering a 380-1050 nm spectral range with 48 bands at a 1-m GSD, Very high resolution RGB imagery at a 5-cm GSD. The image is organized into several separate tiles.

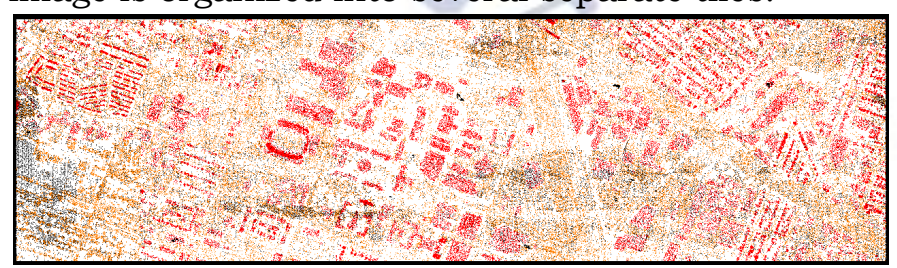

Figure.2, Airborne LiDAR data

\section{METHODOLOGY}

The methodology of the present study includes classification of Lidar point cloud data. For the classification the land cover classes considered are Ground class, low points, Residential buildings, Commercial buildings, Low vegetation, medium vegetation, high vegetation, roads, soil, fly-over, parking lot, metallic objects, stadium, power lines, railway track and open spaces.

\section{Classification of LiDAR point cloud data}

The classification of LiDAR was carried out using Tarascan model. The prior to the classification noise present in the data was removed manually. After the noise removal the ground and non-ground points were classified using ground routine. The non-ground points are further classified into various land cover classes using routines available in the model. Building routine was used to classify residential and commercial buildings. Roads were classified using centerline method, elevation from the ground used to classify vegetation. Likewise, other landcover classes were classified using different routines and manually using terra photo model [17]. The accuracy assessment was carried out in ArcGIS[3].

\section{RESULTS AND DISCUSSION}

In this study Spatial classification of LiDAR data was performed using TERRASCAN using macros containing routines form some classes. The result of classification is shown in fig 3 .,

\section{Classification of LiDAR point cloud data:}

The classified image of LiDAR point cloud data was able to distinguish limited LULC features. Macros with in-built routine classes are used to perform the classification. Some of the classes are manually classified using Terraphoto model. The misclassified points are also corrected in various classes like residential buildings, commercial buildings, high vegetation etc, other than these land cover feature can have performed power line classification. Without manual classification most of the points were misclassified due to the lack of required spectral information.
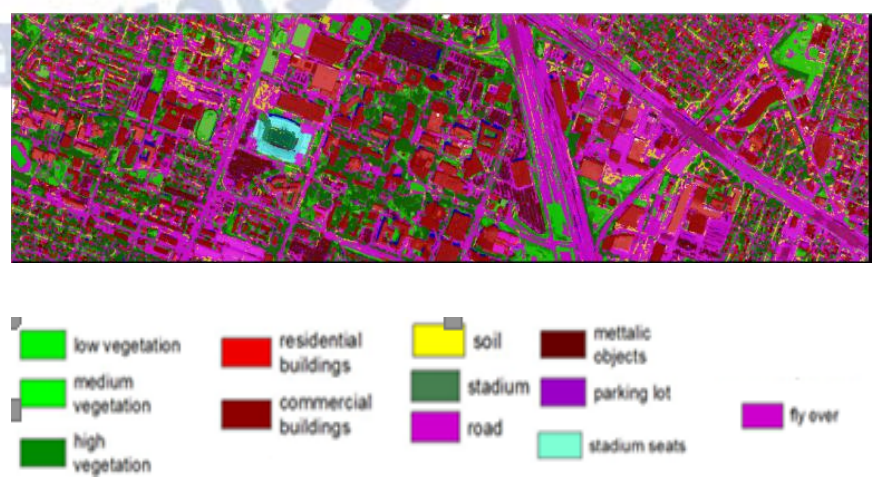
Figure.3,.calassified lidar data

Table1., User and Producers accuracy

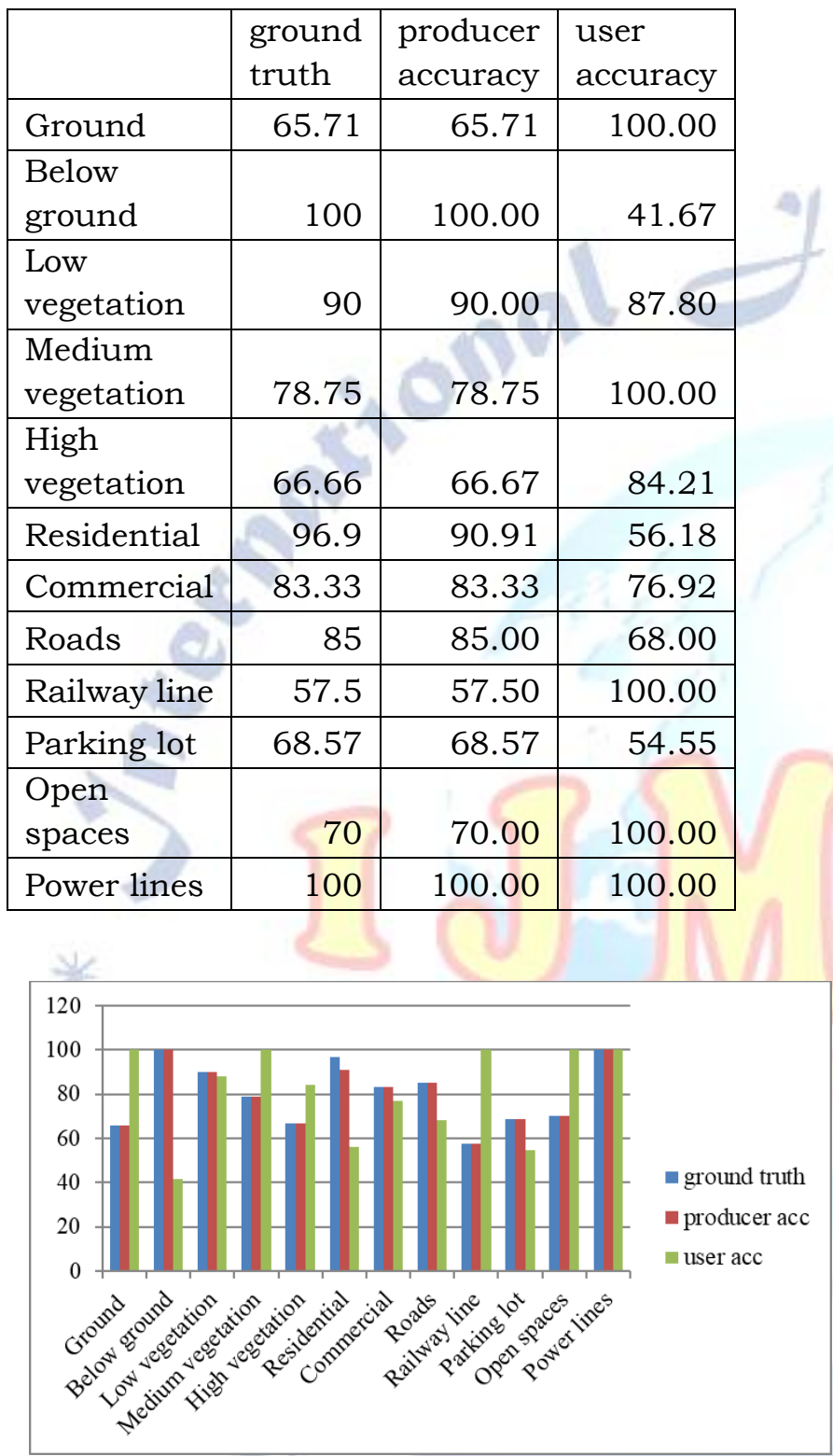

Figure.4, Chart showing producer and user

Table1, presents the Ground truth points, Producer accuracy and User's accuracy. From the chart it is overserved that Producers accuracy for below ground, residential, and power lines are showing maximum accuracy while ground points, medium vegetation, railway lines, open spaces and power lines are showing maximum accuracy in User's accuracy. The overall accuracy of the spatial classification is $85.2 \%$ and kappa coefficient is $0.7562 .$.

\section{CONCLUSION}

This paper presents a work flow for LiDAR point cloud data classification using Terrasoild modules mainly Terrascan. The main goal of this paper is to classify the LiDAR point cloud data into maximum number of classes using only macros available in Terrasoild. Most other previous studies use small number of generic classes like buildings, roads, water bodies etc., While in this study we used 12 classes for finer classification. But the classes like railway lines, open spaces and High vegetation needs more. Our future work will be focusing to Fusion of LiDAR point cloud data and hyperspectral data and classification of integrates data using various methods like Object based classification, SVM classification etc.,

\section{ACKNOWLEDGMENT}

The authors would like to take this opportunity to thank the NSF funded center for Airborne Laser Mapping (NCALM) at the University of Houston for providing data sets for this study through IEEE GRSS data fusion contest.

\section{REFERENCE}

[1] J. S. Rawat and M. Kumar, "Monitoring land use/cover change using remote sensing and GIS techniques: A case study of Hawalbagh block, district Almora, Uttarakhand, India," Egypt. J. Remote Sens. Sp. Sci., vol. 18, no. 1, pp. 77-84, 2015.

[2] R. Verma, K. Kumari, and R. Tiwary, "Application of Remote Sensing and GIS technique for efficient Urban planning in India," Geomatrix Conf. Proc., 2009.

[3] B. Abbasi, H. Arefi, B. Bigdeli, M. Motagh, and S. Roessne, "Fusion of hyperspectral and lidar data based on dimension reduction and maximum likelihood," in International Archives of the Photogrammetry, Remote Sensing and Spatial Information Sciences - ISPRS Archives, 2015.

[4] L. Matikainen, K. Karila, J. Hyyppä, P. Litkey, E. Puttonen, and E. Ahokas, "Object-based analysis of multispectral airborne laser scanner data for land cover classification and map updating," ISPRS J. Photogramm. Remote Sens., vol. 128, pp. 298-313, 2017.

[5] X. Zou, G. Zhao, J. Li, Y. Yang, and Y. Fang, "3D land cover classification based on multispectral lidar point clouds," Int. Arch. Photogramm. Remote Sens. Spat. Inf. Sci. - ISPRS Arch., vol. 2016-Janua, no. July, pp. 741-747, 2016.

[6] N. Ekhtari, C. Glennie, and J. C. Fernandez-Diaz, "Classification of multispectral lidar point clouds," Int. Geosci. Remote Sens. Symp., vol. 2017-July, pp. 2756-2759, 2017.

[7] B. Chen et al., "Multispectral LiDAR point cloud classification: A two-step approach," Remote Sens., vol. 9, no. 4, pp. 1-17, 2017.

[8] B. Höfle, M. Hollaus, and J. Hagenauer, "Urban vegetation detection using radiometrically calibrated small-footprint full-waveform airborne LiDAR data," ISPRS J. Photogramm. Remote Sens., vol. 67, no. 1, pp. 134-147, 2012.

[9] S. A. Panwar, "A Review: Image Fusion Techniques and Applications," vol. 7, no. 3, pp. 406-410, 2015. 
[10] W. Liao, R. Bellens, A. Pizurica, S. Gautama, and W. Philips, "Combining feature fusion and decision fusion for classification of hyperspectral and LiDAR data," Int. Geosci. Remote Sens. Symp., pp. 1241-1244, 2014.

[11] S. Luo et al., "Fusion of airborne discrete-return LiDAR and hyperspectral data for land cover classification," Remote Sens., vol. 8, no. 1, 2016.

[12] P. Ghamisi, J. A. Benediktsson, and S. Phinn, "Fusion of hyperspectral and LiDAR data in classification of urban areas," Int. Geosci. Remote Sens. Symp., pp. 181-184, 2014.

[13] B. Rasti, P. Ghamisi, J. Plaza, and A. Plaza, "Fusion of Hyperspectral and LiDAR Data Using Sparse and Low-Rank Component Analysis," IEEE Trans. Geosci. Remote Sens., vol. 55, no. 11, pp. 6354-6365, 2017.

[14] A. Behan, "On the matching accuracy of rasterised scanning laser altimeter data," Int. Arch. Photogramm. Remote Sens., 2000.

[13] J. P. M. O'Neil-Dunne, S. W. MacFaden, A. R. Royar, and K. C. Pelletier, "An object-based system for LiDAR data fusion and feature extraction," Geocarto Int., vol. 28, no. 3, pp. 227-242, 2013.

[14] a Ono, K. Kajiwara, and Y. Honda, "Development of New Vegetation Indexes, Shadow Index ( Si ) and Water Stress Trend (Wst )," Int. Arch. Photogramm. Remote Sens. Spat. Innformation Sci., vol. XXXVIII, pp. 710-714, 2010.

[15] J. A. Gamon, L. Serrano, and J. S. Surfus, "The photochemical reflectance index: An optical indicator of photosynthetic radiation use efficiency across species, functional types, and nutrient levels," Oecologia, 1997.

[16] M. Fauvel, J. A. Benediktsson, J. Chanussot, J. R. Sveinsson, and J. A. Benediktsson, "Spectral and Spatial Classification of Hyperspectral Data Using SVMs and Morphological Profiles Spectral and Spa-tial Classification of Hyperspectral Data Using SVMs and Morphological Profiles. IEEE Transactions on Geoscience and Remote Sensing, Institute," Electron. Eng., vol. 46, no. 11, pp. 3804-3814, 2008.

[17] C. I. Miller, J. J. Thomas, A. M. Kim, J. P. Metcalf, and R. C. Olsen, "Application of image classification techniques to multispectral lidar point cloud data," Laser Radar Technol. Appl. XXI, vol.

9832, p. 98320X, 2016.

[18] M. Khodadadzadeh, J. Li, S. Prasad, and A. Plaza, "Fusion of Hyperspectral and LiDAR Remote Sensing Data Using Multiple Feature Learning," IEEE J. Sel. Top. Appl. Earth Obs. Remote Sens., vol. 8, no. 6, pp. 2971-2983, 2015.

[19] P. Ghamisi, R. Souza, J. A. Benediktsson, L. Rittner, R. Lotufo, and X. X. Zhu, "Hyperspectral data classification using extended extinction profiles," IEEE Geosci. Remote Sens. Lett., vol. 13, no. 11, pp. 1641-1645, 2016.
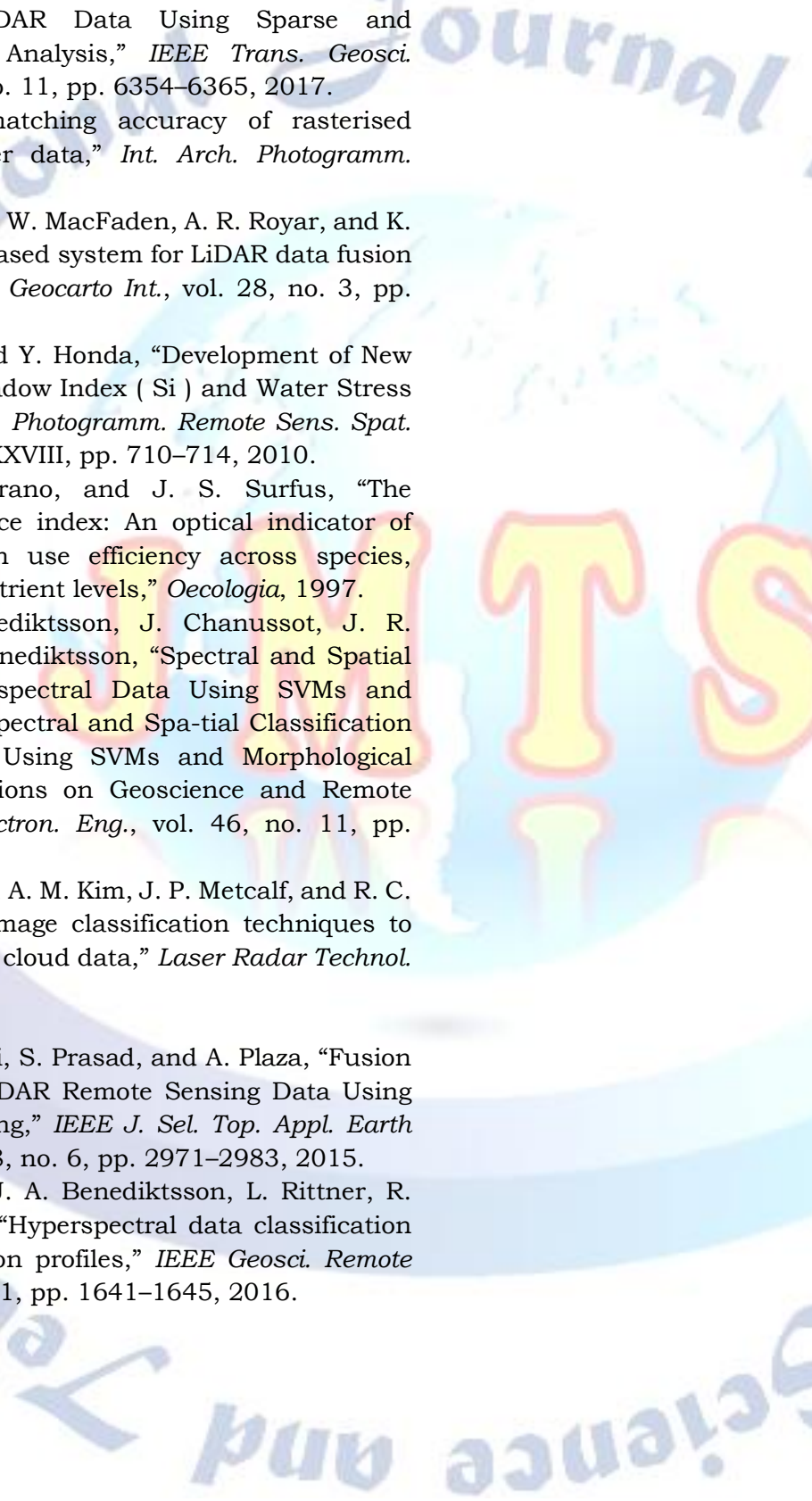